\title{
Structure of $X_{b}$ from line shape analysis
}

\author{
Yue $\mathrm{Ma}^{1}$, Guo-Ying Chen $^{1,2}$ \\ 1) Department of Physics, Xinjiang University, Urumqi 830046, China and \\ 2) State Key Laboratory of Theoretical Physics, Institute of Theoretical Physics, \\ Chinese Academy of Sciences, Beijing 100190, China
}

(Dated: May 6, 2019)

\begin{abstract}
We study the production line shape of $B^{*} \bar{B}$ near threshold, where the $B^{*} \bar{B}$ pair comes from the resonance $X_{b}$. Our study shows that the line shape depends sensitively on the binding energy and the probability of finding an elementary state in the physical bound state. Both of the two parameters are crucial to identify the structure of $X_{b}$. Therefore, the line shape measurement can shed light on the structure of $X_{b}$.

PACS numbers:
\end{abstract}

The discovery of $X(3872)$ by the Belle collaboration in 2003 [1] has initiated tremendous interest in both experimental and theoretical studies. From then on, many "exotic" mesons have been discovered, and they are generally called XYZ states. The nature of $X(3872)$ is still under debate, although it was discovered more than ten years ago. As the mass of $X(3872)$ is close to $D^{0 *} \bar{D}^{0}$ threshold, $X(3872)$ is expected to be a candidate for a hadron molecular state [2]. However, the large production rates of $X(3872)$ in the B-factories and at the Tevatron seem to favor a compact structure in its wave function. Taking these facts into account, it seems reasonable to identify $X(3872)$ as a mixing state between a $J^{P C}=1^{++} c \bar{c}$ component and a $D^{0 *} \bar{D}^{0}$ component $[3,4]$. Obviously, more experimental data and theoretical developments are required to clarify the nature of $X(3872)$. It has been proposed that the search for the bottomonium counterpart of $X(3872)$, which is usually called $X_{b}$, may shed light on the structure of $X(3872)$. However, no clear evidence for the existence of such a state has been found up to now $[5,6]$.

Inspired by the assignment that $X(3872)$ can be a mixture of $c \bar{c}$ and $D^{0 *} \bar{D}^{0}$, Ref. [7] proposed to identify the already discovered $\chi_{b 1}(3 P)[8-11]$ as $X_{b}$. As demonstrated in [7], a key feature of $\chi_{b 1}(3 P)$ is the dominance of radiative decay to $\gamma \Upsilon(3 S)$ over $\gamma \Upsilon(2 S)$ or $\gamma \Upsilon(1 S)$. To confirm the discovery of $X_{b}$, one also needs to show that this state has an essential component of an $S$-wave $B^{*} \bar{B}$ molecule. Actually, this is the main purpose of the present work. We use the notation $B^{*} \bar{B}$ to denote $B^{* 0} \bar{B}^{0}, B^{0} \bar{B}^{* 0}, B^{+} \bar{B}^{*-}$ and $B^{-} \bar{B}^{*+}$.

The single-channel potential model predicts that the mass of $\chi_{c 1}(2 P)$ is around $3950 \mathrm{MeV}$ [12]. The expected relation between three $\chi_{c}(2 P)$ states is

$$
m_{\chi_{c 0}(2 P)}<m_{\chi_{c 1}(2 P)}<m_{\chi_{c 2}(2 P)} .
$$

The absence of $\chi_{c 1}(2 P)$ around $3950 \mathrm{MeV}$ and the discovery of the puzzling charmonium-like state $X(3872)$ make the hadron spectrum a very interesting topic. It is worth mentioning that the single-channel prediction of $\chi_{c 1}(2 P)$ mass should be viewed with some caution, since coupled-channel effects can modify such a 
prediction significantly [12]. Identifying $X(3872)$ as a mixing state between $\chi_{c 1}(2 P)$ and a $D^{0 *} \bar{D}^{0}$ molecule can explain the missing $\chi_{c 1}(2 P)$ around $3950 \mathrm{MeV}$ and also make the relation $m_{X(3872)}<m_{\chi_{c 0}(2 P)}<$ $m_{\chi_{c 2}(2 P)}$ easy to understand, as the mass of $\chi_{c 0}(2 P)$ lies above $D^{0 *} \bar{D}^{0}$ threshold.

Similar to $X(3872)$, the mass of $X_{b}$ should be sensitive to its structure. Present experimental results cannot give the mass splittings between different $\chi_{b}(3 P)$ states. By assuming a mass splitting between $\chi_{b 1}(3 P)$ and $\chi_{b 2}(3 P)$ states, i.e., $\Delta m_{12}=m_{\chi_{b 2}(3 P)}-m_{\chi_{b 1}(3 P)}$, to be $10.5 \mathrm{MeV}$, LHCb gives $m_{\chi_{b 1}(3 P)}=10515.7_{-3.9-2.1}^{+2.2+1.5} \mathrm{MeV}$ [11]. This value is close to the early calculation with a potential model, in which $m_{\chi_{b 1}(3 P)}=10516 \mathrm{MeV}$ [13]. Nevertheless, one should note that if the discovered $\chi_{b 1}(3 P)$ state is a counterpart of $X(3872)$, it is possible that its mass may be larger than that of $\chi_{b 2}(3 P)$, since $X_{b}$ contains a substantial $B^{*} \bar{B}$ component, and the $B^{*} \bar{B}$ threshold is around $10605 \mathrm{MeV}$. A realistic unquenched quark model calculation predicts $m_{\chi_{b 1}(3 P)}=10580 \mathrm{MeV}$, while $m_{\chi_{b 2}(3 P)}=10578 \mathrm{MeV}$ [14].

As the main purpose of the present work is to study the line shape of $B^{*} \bar{B}$ near threshold within an effective field theory (EFT), we would first introduce the EFT approach developed in [15, 16]. Consider a bare state $|\mathcal{B}\rangle$ with bare mass $\left(-B_{0}\right)$ and coupling $g_{0}$ to the two-particle state, where the bare mass is defined relative to the two-particle threshold. The two particles have masses $m_{1}, m_{2}$ respectively. If $|\mathcal{B}\rangle$ is near the two-particle threshold, then the leading two-particle scattering amplitude can be obtained by summing the Feynman diagrams in Fig. 1. Near the threshold, the three-momenta of the two particles are non-relativistic. With the minimal subtraction (MS) scheme, the loop integral can be written as

$$
\begin{aligned}
\mathcal{I}^{M S} & \equiv \int \frac{d^{D} \ell}{(2 \pi)^{D}} \frac{i}{\left[\ell^{0}-\overrightarrow{\ell^{2}} /\left(2 m_{1}\right)+i \epsilon\right]} \cdot \frac{i}{\left[E-\ell^{0}-\vec{\ell}^{2} /\left(2 m_{2}\right)+i \epsilon\right]} \\
& =i \frac{\mu}{2 \pi} \sqrt{-2 \mu E-i \epsilon},
\end{aligned}
$$

where $\mu$ is the reduced mass of the two particles, and $E$ is the kinematic energy of the two-particle system. We then have the two-body elastic scattering amplitude for Fig. 1

$$
\mathcal{A}=-\frac{g_{0}^{2}}{E+B_{0}-g_{0}^{2} \frac{\mu}{2 \pi} \sqrt{-2 \mu E-i \epsilon}} .
$$

Because a physical bound state with binding energy $\mathrm{B}$ corresponds to a pole at $E=-B$, we have

$$
B_{0}=B+g_{0}^{2} \frac{\mu}{2 \pi} \sqrt{2 \mu B}
$$

Expanding Eq. (3) around the pole, one obtains

$$
\mathcal{A}=-\frac{\delta}{E+B+\Sigma(E)}
$$

where

$$
\delta=\frac{g_{0}^{2}}{1+g_{0}^{2} \mu^{2} /(2 \pi \sqrt{2 \mu B})}, \quad \Sigma(E)=-\delta\left[\frac{\mu}{2 \pi} \sqrt{-2 \mu E-i \epsilon}+\frac{\mu \sqrt{2 \mu B}}{4 \pi B}(E-B)\right] .
$$

In Ref. $[17,18]$, Weinberg showed that, in the leading approximation, the coupling $g$ between a physical bound state and the $S$-wave two-particle state satisfies

$$
g^{2}=\frac{2 \pi \sqrt{2 \mu B}}{\mu^{2}}(1-Z)
$$


where $Z$ is the probability of finding an elementary state in the physical bound state. Eq. (7) can be connected to the framework of the EFT through

$$
\delta=g^{2} .
$$

This connection leads to the following relations

$$
g_{0}^{2}=g^{2} / Z, \quad B_{0}=\frac{2-Z}{Z} B .
$$

With Eq. (9), Eq. (3) can be re-expressed as

$$
\mathcal{A}=-\frac{g^{2}}{E+B+\tilde{\Sigma}(E)},
$$

where

$$
\tilde{\Sigma}(E)=-g^{2}\left[\frac{\mu}{2 \pi} \sqrt{-2 \mu E-i \epsilon}+\frac{\mu \sqrt{2 \mu B}}{4 \pi B}(E-B)\right] .
$$

We can also express Eq. (10) in the form

$$
i \mathcal{A}=i g_{0} \cdot G(E) \cdot i g_{0},
$$

where $G(E)$ is the complete propagator for the $S$-wave near-threshold state

$$
G(E)=\frac{i Z}{E+B+\tilde{\Sigma}(E)+i \Gamma / 2},
$$

and we have included the width $\Gamma$ in the propagator. This width comes from the decay modes which do not proceed through the molecular component. From Eq. (12), we find that the Feynman rule for the coupling between the near-threshold state and the two-particle state can be written as $i g_{0}$. Treating the binding momentum $\gamma=(2 \mu B)^{1 / 2}$ and the three-momentum of the two-particle state $p$ as small scales, i.e., $\gamma, p \sim \mathcal{O}(p)$, one can find that the leading amplitude Eq. (10) is at the order of $\mathcal{O}\left(p^{-1}\right)$.

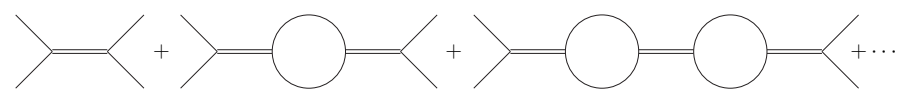

FIG. 1: Feynman diagrams for the two particle scattering. The double lines denote the bare state.

Now we come to study the line shape of $B^{*} \bar{B}$ near threshold. As $X_{b}$ contains essential $B^{*} \bar{B}$ molecular component, its coupling with the $B^{*} \bar{B}$ pair is large. Therefore, one can expect that the near threshold production of the $B^{*} \bar{B}$ pairs mainly comes from the intermediate $X_{b}$, see Fig. 2.

With the EFT approach proposed in [15], we can write out the amplitude for Fig. 2 directly

$$
i \mathcal{M}=\mathcal{A}_{X_{b}} \cdot \frac{i Z}{E+B+\tilde{\Sigma}(E)+i \Gamma / 2} \cdot \frac{i g_{0}}{2},
$$




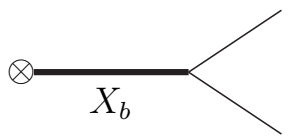

FIG. 2: Feynman diagram for $X_{b} \rightarrow B^{*} B$. The cross denotes the production vertex of $X_{b}$.

where $\mathcal{A}_{X_{b}}$ denotes the production vertex of $X_{b}, B$ is the binding energy, $\mu$ is the reduced mass of $B^{*} \bar{B}, Z$ is the probability of finding $\chi_{b 1}(3 P)$ component in $X_{b}$, and $\Gamma$ is the width which probably comes from the decay of $\chi_{b 1}(3 P)$. One can find that this amplitude is proportional to $\sqrt{Z(1-Z)}$. Therefore, if $Z=0$ or $Z=1$, this amplitude vanishes. It is not difficult to understand this behavior, as $X_{b}$ is produced through its compact component $b \bar{b}$. After its production, the $b \bar{b}$ pair will evolve into $B^{*} \bar{B}$ through the coupled channel effect. We assume that $\mathcal{A}_{X_{b}}$ is not sensitive to the energy, so it can be treated as a constant.

Using the amplitude given in Eq. (14), one can plot the line shape of the $B^{*} \bar{B}$. As the mass of $X_{b}$ is not yet settled, we choose two different values: one is $m_{X_{b}}=10515.7 \mathrm{MeV}$ [11] which is the recent measured value by $\mathrm{LHCb}$, and the other is $m_{X_{b}}=10580 \mathrm{MeV}$ [14] which is the prediction of an unquenched quark model. We set the width $\Gamma=0$, considering the fact that $X(3872)$ is a very narrow state. We show the line shape in Fig. 3 and Fig. 4 . One can see that the line shape depends sensitively on $Z$. If the mass of $X_{b}$ is close to the lower value $m_{X_{b}}=10515.7 \mathrm{MeV}$, the near threshold enhancement would not be clear. One can also find that the near threshold enhancement can be more significant for a larger $Z$. We have used an arbitrary normalization in the figure.

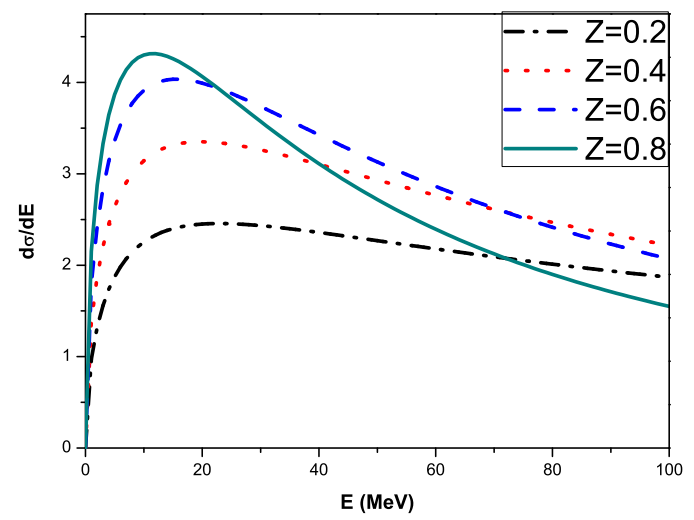

FIG. 3: Line shape of $B^{*} \bar{B}$ with $m_{X_{b}}=10580 \mathrm{MeV}$.

For comparison, we come to the special case where $X_{b}$ is a pure $B^{*} \bar{B}$ molecule or $Z=0$. As was pointed out previously, Eq. (14) vanishes for $Z=0$. If $X_{b}$ is a pure $B^{*} \bar{B}$ molecule, its production should proceed via $B^{*} \bar{B}$ rescattering as shown in Fig. 5 . It is not difficult to show that the non-resonant background term 


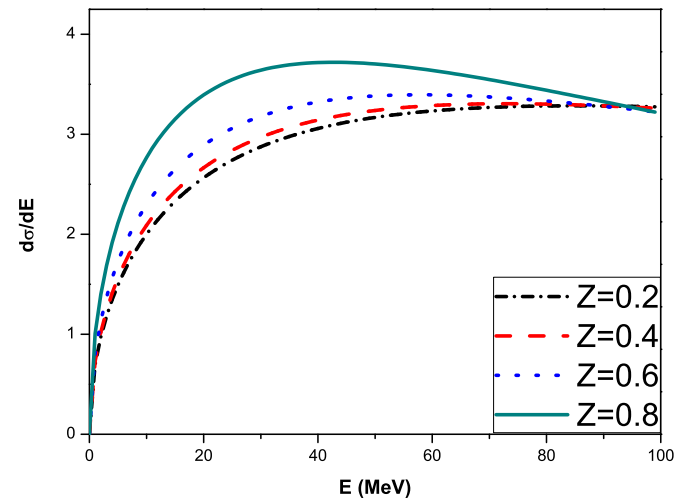

FIG. 4: Line shape of $B^{*} \bar{B}$ with $m_{X_{b}}=10515.7 \mathrm{MeV}$

contributes at the same order as that in Fig. 5 (one can refer to Ref. [15] for details). Thus at the leading order, one should use the Feynman diagrams as shown in Fig. 6. The amplitude for Fig. 6 can be written as

$$
i \mathcal{M}_{Z=0}=\mathcal{A}_{B \bar{B}^{*}} \frac{2 B+i \Gamma / 2}{2 B-\sqrt{2 B / \mu} \cdot \sqrt{-2 \mu E-i \epsilon}+i \Gamma / 2}
$$

where $\mathcal{A}_{B \bar{B}^{*}}$ denotes the first production vertex in Fig. 5. Near the threshold, we can treat $\mathcal{A}_{B \bar{B}^{*}}$ as a constant. We use the binding energy $B=0.18 \mathrm{MeV}$, which was predicted in [19]. We then show the corresponding line shape of Eq. (15) in Fig. 7 as a dashed line. The dashed-dotted line in Fig. 7 denotes the resonant contribution of Fig. 5. The solid line in Fig. 7 is the line shape of Eq. (14) with $Z=0.2$ and the binding energy chosen to be $B=0.18 \mathrm{MeV}$. Two conclusions can be obtained from Fig. 7:

- By comparing the dashed line and the dashed-dotted line, one can find that in the pure molecule scenario the near threshold enhancement only appears when the non-resonant background term is taken into account. Hence it seems necessary to consider the non-resonant background in the pure molecule scenario.

- By comparing the solid line and the dashed line, one can see that the near threshold enhancement appears in both the pure molecule scenario and the scenario in which the near-threshold state contains a substantial compact component. It is interesting to notice that with the same binding energy, the near threshold enhancement is more significant in the latter scenario.

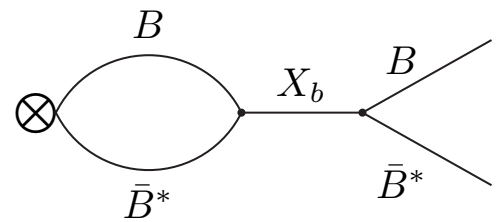

FIG. 5: Feynman diagram of leading resonant contribution in the special case $Z=0$. 


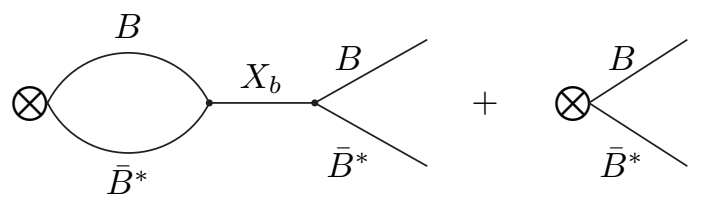

FIG. 6: Feynman diagrams of all leading contributions in the special case $Z=0$.

In summary, we have used the effective field theory which was developed in Ref. $[15,16]$ to study the line shape of $B^{*} \bar{B}$ near threshold. It is shown that the line shape depends sensitively on two parameters, i.e., the probability of finding a compact component in the physical bound state $Z$ and the binding energy $B$. Both of these two parameters are important for understanding the structure of $X_{b}$. Therefore, the line shape measurement can help us to identify the structure of $X_{b}$.

ACKNOWLEDGMENTS: We would like to thank Wen-Sheng Huo for a careful reading of the manuscript and valuable comments. This work is supported, in part, by National Natural Science Foundation of China (Grant Nos. 11147022 and 11305137) and Doctoral Foundation of Xinjiang University (No. BS110104). Yue Ma is also supported by National Natural Science Foundation of China (Grant No.11203018).

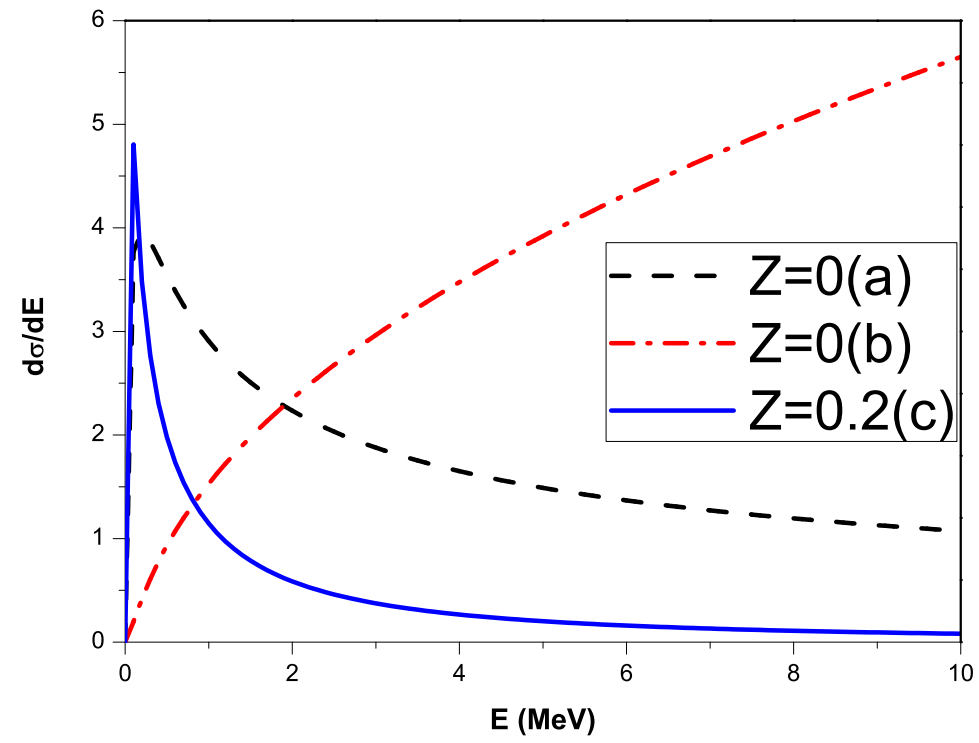

FIG. 7: Line shape of $B^{*} \bar{B}$. (a)Dashed line is the line shape predicted by Eq. (15). (b) Dashed-dotted line denotes the resonant contribution of Fig. 5. (c) Solid line is the line shape predicted by Eq. (14) with $B=0.18 \mathrm{MeV}$ and $Z=0.2$.

[1] S. K. Choi et al. [Belle Collaboration], Phys. Rev. Lett. 91, 262001 (2003) [hep-ex/0309032]. 
[2] N. A. Tornqvist, Phys. Lett. B 590, 209 (2004) [hep-ph/0402237].

[3] C. Meng, Y. J. Gao and K. T. Chao, Phys. Rev. D 87, no. 7, 074035 (2013) [hep-ph/0506222].

[4] M. Suzuki, Phys. Rev. D 72, 114013 (2005) [hep-ph/0508258].

[5] G. Aad et al. [ ATLAS Collaboration], arXiv:1410.4409 [hep-ex].

[6] X. H. He et al. [Belle Collaboration], Phys. Rev. Lett. 113, 142001 (2014) [arXiv:1408.0504 [hep-ex]].

[7] M. Karliner and J. L. Rosner, Phys. Rev. D 91, 014014 (2015) [arXiv:1410.7729 [hep-ph]].

[8] G. Aad et al. [ATLAS Collaboration], Phys. Rev. Lett. 108, 152001 (2012) [arXiv:1112.5154 [hep-ex]].

[9] V. M. Abazov et al. [D0 Collaboration], Phys. Rev. D 86, 031103 (2012) [arXiv:1203.6034 [hep-ex]].

[10] R. Aaij et al. [LHCb Collaboration], Eur. Phys. J. C 74,3092 (2014) [arXiv:1407.7734 [hep-ex]].

[11] R. Aaij et al. [LHCb Collaboration], JHEP 1410, 88 (2014) [arXiv:1409.1408 [hep-ex]].

[12] I.V.Danilkin and Y.A.Simonov, Phys. Rev. Lett.105, 102002 (2010)

[13] W. Kwong and J. L. Rosner, Phys. Rev. D 38, 279 (1988).

[14] J. Ferretti and E. Santopinto, Phys. Rev. D 90, 094022 (2014) [arXiv:1306.2874 [hep-ph]].

[15] G. Y. Chen, W. S. Huo and Q. Zhao, Chinese Physics C 39, No.9, 093101 (2015) [arXiv:1309.2859 [hep-ph]].

[16] W. S. Huo and G. Y. Chen, arXiv:1501.02189 [hep-ph].

[17] S. Weinberg, Phys. Rev. 130, 776 (1963).

[18] S. Weinberg, Phys. Rev. 137, B672 (1965).

[19] M. T. AlFiky, F. Gabbiani and A. A. Petrov, Phys. Lett. B 640, 238 (2006) [hep-ph/0506141]. 